\title{
Immediate Induction and Consolidation Therapy May Improve Outcomes for Patients with Anaplastic Astrocytoma: a Case Series
}

\author{
Jonathan D. Santoro, ${ }^{1, *}$, Julie Kanter ${ }^{2}$ \\ ${ }^{1}$ Lucille Packard Children’s Hospital at Stanford University School of Medicine, Department of Pediatrics, Palo Alto, CA \\ ${ }^{2}$ Medical University of South Carolina, Department of Pediatrics, Division of Hematology-Oncology, Charleston, SC \\ *Corresponding author: jsantoro@stanford.edu
}

Received February 21, 2014; Revised March 03, 2014; Accepted March 06, 2014

\begin{abstract}
Despite advances in chemotherapy and radiation, prognosis for all patients with high grade gliomas remains poor. In anaplastic astrocytomas (WHO III) GTR is associated with a 5 year progression free survival (PFS) of $44 \%$ compared to $22 \%$ when surgical resection is sub-total. While initial studies demonstrated post-radiation chemotherapy improved outcomes, successive chemotherapeutic trials were unable to significantly improve outcomes. Recent trials in adults with recurrent glioblastoma multiforme have shown that the combination of Bevacixumab and Irinotecan have improved 6 month PFS was 46\% and overall survival (OS) at 6 months of 77\%. Although these adult studies were encouraging, results were not replicated in children with similar pathology. This case series presents two pediatric patients with anaplastic astrocytoma treated with an induction therapy of Temozolamide (90mg/m2 x 42 days) plus standard radiation therapy (5400 cGy) very shortly after surgical resection, followed by a consolidation treatment regimen of 8-cycles of chemotherapy with Bevacizumab $(10 \mathrm{mg} / \mathrm{m} 2)$ and Irinotecan $(125 \mathrm{mg} / \mathrm{m} 2)$, given on days 1 and 15 of 21 day cycles. These patients did extraordinarily well on this therapeutic regimen, surpassing expectations, with one patient having 18 months of PFS and one patient achieving CR1 at the time of this publication for 20 months. The treatment regimen was well tolerated without unanticipated toxicities or episodes of severe neutropenia induced by this therapy. We are encouraged by the improvements in PFS and OS in these two patients as well as the acceptability of toxicity in this case series and thus recommend prompt induction and consolidation chemotherapy following resection of these neoplasms.
\end{abstract}

Keywords: high grade astrocytoma, chemotherapy, tumor, brain, bevacizumab, irinotecan

Cite This Article: Jonathan D. Santoro, Julie Kanter, "Immediate Induction and Consolidation Therapy May Improve Outcomes for Patients with Anaplastic Astrocytoma: a Case Series." American Journal of Medical Sciences and Medicine, vol. 2, no. 2 (2014): 37-40. doi: ajmsm-2-2-2.

\section{Background}

Primary brain tumors derived from oligodendroglia or astrocytes are known as gliomas. The World Health Organization (WHO) has classified these tumors into low grade astrocytomas, which are grades I or II, and high grade astrocytomas, which are grades III or IV [11]. WHO classification is primarily performed on biopsy specimens where anaplastic features and high mitotic activity are noted with or without the presence of vascular proliferation and/or necrosis. High grade gliomas (HGG) are more common in adults but also comprise $3-7 \%$ of primary brain tumors in children and typically occur in early to late adolescence [9,17]. There are several associations between the development of these types of tumors and rare inherited tumor predisposition syndromes such as Li Fraumeni syndrome, Neurofibromatosis type I, and Turcott syndrome. There are no other known risk factors to development of HGG outside of prior radiation exposure $[6,10,18,20]$.
Despite advances in chemotherapy and radiation, prognosis for all patients with HGG remains poor. Standard of care in the treatment of HGG includes gross total surgical resection (GTR) followed by targeted external beam radiation (when patients are above 2 years of age) and/or chemotherapy. Surgical intervention is largely influenced by the location of the malignancy as well as the minimization of severe neurological complications. In anaplastic astrocytomas (WHO III) GTR is associated with a 5 year progression free survival (PFS) of $44 \%$ compared to $22 \%$ when surgical resection is subtotal [21].

The role of chemotherapy after neurosurgical resection in anaplastic astrocytoma remains unclear. Initial trials by the Children's Cancer Group (CCG) in children with HGG treated with post-radiation chemotherapy (vincristine, lomustine and predinose) had an improved progression free survival (PFS) of $46 \%$ compared to $26 \%$ of those treated with radiation alone [15].Despite these initial findings, successive chemotherapeutic trials were unable to significantly improve outcomes [3,11]. Promising data has been published in adult patients with 
HGG treated with the methylating agent Temozolamide when administered with concurrent radiation therapy, suggesting a role for adjuvant chemotherapy in pediatric patients as well although subsequent trials have been slow to demonstrate similar improvement [1]. Recent trials in adults with recurrent glioblastoma multiforme have shown that the combination of Bevacixumab and Irinotecan have improved 6 month PFS was $46 \%$ and overall survival (OS) at 6 months of $77 \%$ [19]. While these results were encouraging in adults, a similar trial of Bevacizumab (10 $\mathrm{mg} / \mathrm{kg})$ and Irinotecan $\left(125 \mathrm{mg} / \mathrm{m}^{3}\right)$ in children with recurrent glioblastoma multiforme who had previously received Temozolamide as initial therapy yielded mixed results with a PFS of just $18 \%$ and an OS of $58.3 \%$ at 6 months. In addition to lack of clinical improvement, this study also documented severe toxicities to chemotherapy which included intra-tumoral hemorrhage, deep vein thrombosis, anaphalaxis, skin rash, and delayed wound healing [8,19].

Currently, a study investigating administration of temodar and radiotherapy (after initial resection) followed by bevacizumab and irinotecan is underway by the Children's Oncology Group under the code "COG ACNS0822”. The study is designed to enroll over 100 patients with HGG to one of three treatment arms. While the results of this study will not be available for some time, it is our goal to stress the importance of immediate consultation with oncology and radiation oncology upon discovery of HGG. Here, we present two pediatric patients with anaplastic astrocytoma treated with an immediate induction therapy of Temazolamide (90mg/m2 x 42 days) plus standard radiation therapy (5400 cGy) followed by an immediate consolidation treatment regimen of 8-cycles of chemotherapy with Bevacizumab (10mg/m2) and Irinotecan (125mg/m2), given on days 1 and 15 of 21 day cycles. As discussed below, their results were significantly superior than young patients with HGG treated with similar regimens or just resection.

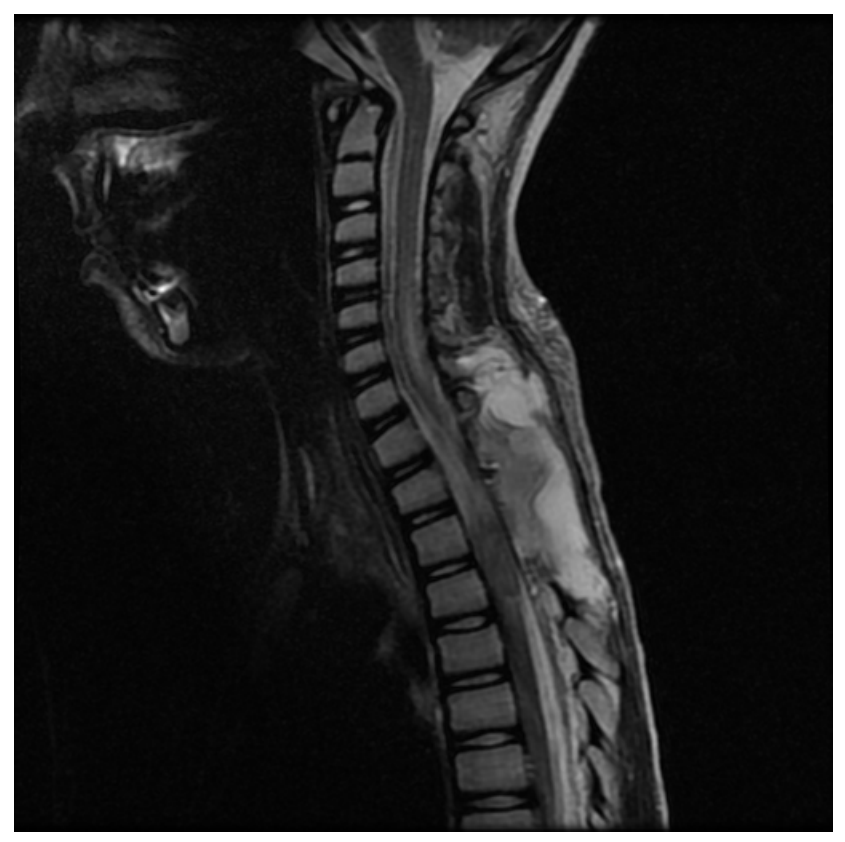

Figure 1. Sagital SE TOP at Admit

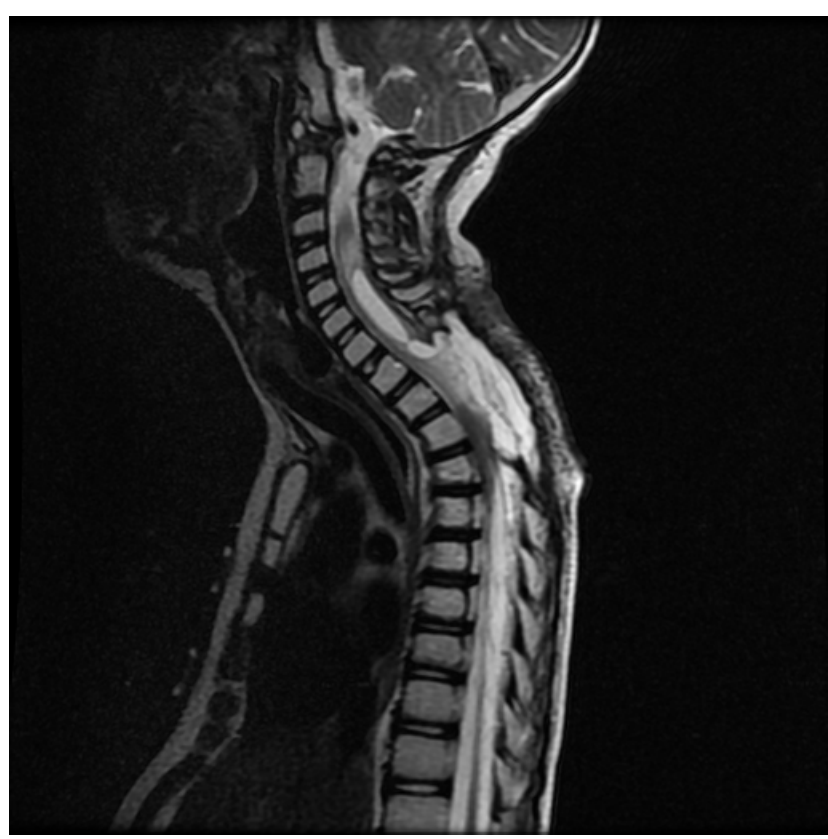

Figure 2. Sagital T1 SE TOP at 3 months post-operative

\subsection{Case I}

HL, a previously healthy 8-year old caucasian female was admitted with a history of severe upper back pain and occasional numbness in her bilateral lower extremities but without additional focalizing neurologic symptoms. Magnetic resonance imaging (MRI) demonstrated an intrathecal spinal cord mass at the level of T2-T4 with a syringomyelia at the level of T1-T2 (Figure 1). The mass was found to be heterogeneously enhancing and spanned the entire area of the spinal cord at that level. The patient underwent a T1-T4 laminectomy, decompression of the syrinx, and biopsy of the mass. The neoplasm could not be fully resected due to its placement within and around the spinal cord (Figure 2). Biopsy results confirmed WHO grade III anaplastic astrocytoma.

Post-operatively the patient did well and was neurologically intact, with complete resolution of the back pain she had reported prior to admission. Radiotherapy and concurrent Temozolamide $\left(90 \mathrm{mg} / \mathrm{m}^{2}\right.$ daily for 42 days) was begun 6 weeks following her initial surgery. Four weeks after completion of this induction therapy, she was started on consolidation therapy with Bevacizumab $(10 \mathrm{mg} / \mathrm{kg})$ and Irinotecan $(125 \mathrm{mg} / \mathrm{m} 2)$ given on days 1 and 15 of a 21 day cycle for eight cycles.

The treatment was well tolerated with only minimal side effects of constipation and occasional nausea. There were no sustained periods of neutropenia or serious infections. Her only major toxicity was severe kyphoscoliosis as a result of her laminectomy which later required surgical intervention. She was in complete remission (CR1) at the completion of therapy and remained in remission for 18 months post treatment until she presented with paraplegia. At that time, imaging could not be performed due to the kyphoscoliosis repair, thus she was noted to have relapsed disease on surgical exploration, which demonstrated tumor invading the spinal cord from T1-T5.

\subsection{Case II}


$\mathrm{AB}$, a previously healthy 3-year old caucasian female, presented with a 2 month history of worsening left arm and leg weakness, abnormal gait, and 2-days of morning emesis and severe headache. MRI revealed a 4.0 x 3.8 x $4.4 \mathrm{~cm}$ rim enhancing mass along the border of the right basal ganglia and thalamus with central hypointensity causing midline shift to the left of approximately $6 \mathrm{~mm}$ (Figure 3). She underwent a craniotomy with gross total resection (Figure 4). Pathology was determined to be WHO grade III anaplastic astocytoma.

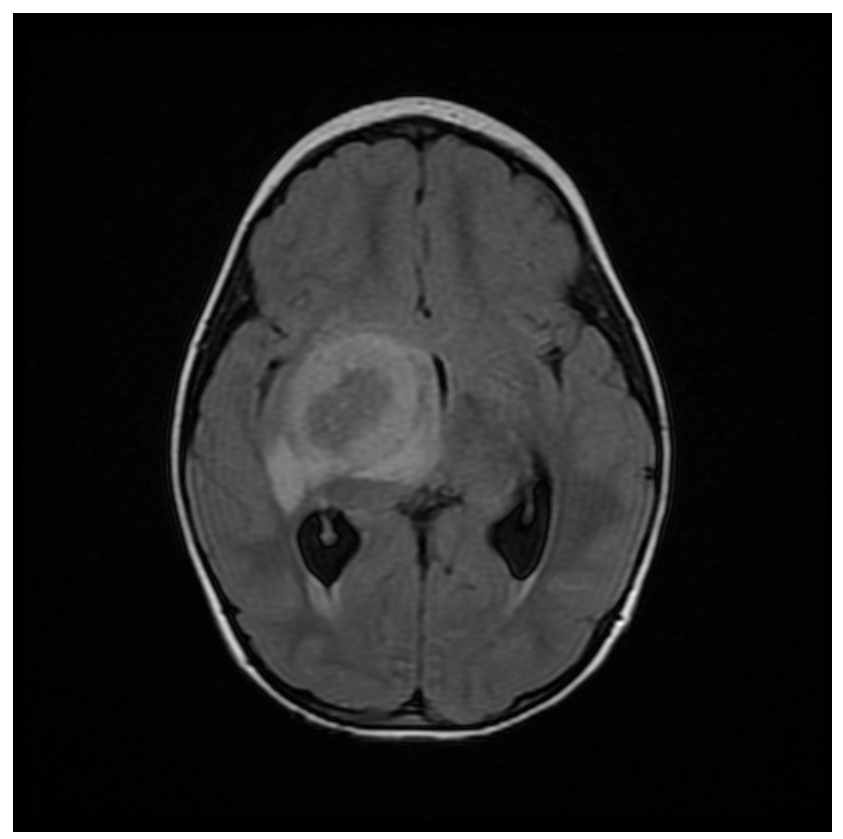

Figure 3. Axial T2 Flair at Admit

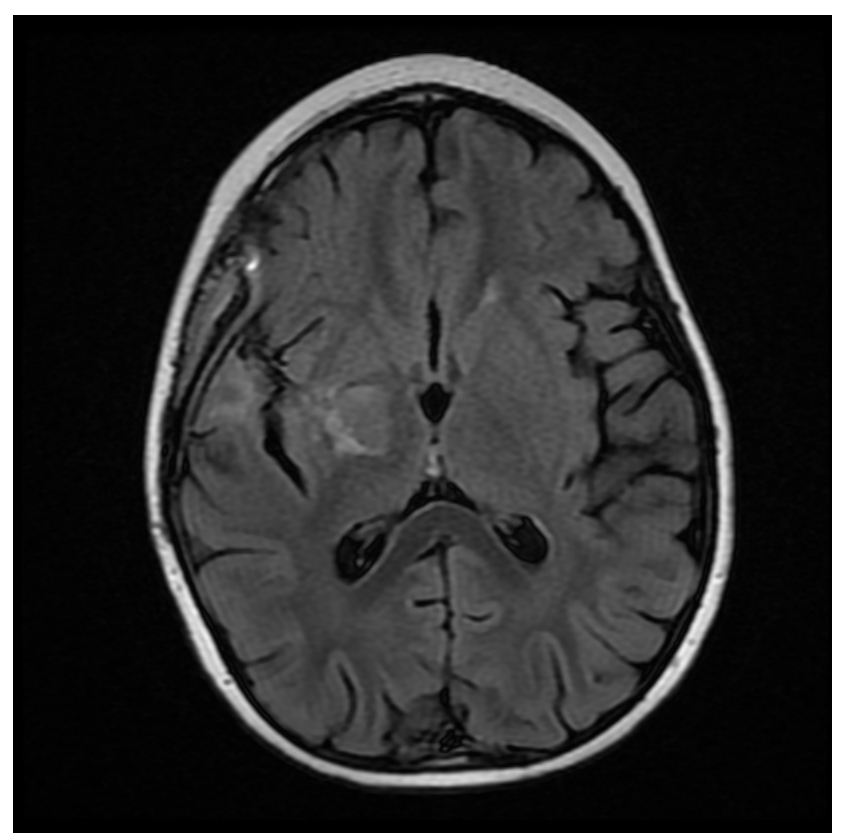

Figure 4. Axial T2 Flair at 6 months post-operative

Post-operatively the patient did well except for persistent hemiplegia (3/5 strength in her left arm, hand, and leg). Radiation therapy (total of 54 Gy) and concomitant Temozolamide ( $90 \mathrm{mg} / \mathrm{m}^{2}$ for 42 days) was initiated 4 weeks following surgery. She tolerated this induction therapy well and was in CR1 at the end of induction. Following a 4-week break, she received consolidation therapy with 8 cycles of Bevacizumab (10mg/kg/dose) and Irinotecan (125mg/m2 dose) given on days 1 and 15 of a 21-day cycle. Her chemotherapy was complicated by occasional nose bleeds, nausea, vomiting, diarrhea, and constipation. She had no serious infections or neutropenic periods.

The patient has remained in full CR-1 for 20 months with continued improvement in left sided strength and mobility. She has had no long-term side effects from her chemotherapy.

\section{Discussion}

Despite the general advances in chemotherapeutic management of many solid tumors, outcomes with high grade astrocytoma continues to remain poor. While initial trials by the CCG demonstrated improvements in PFS in patients treated with a combination of chemotherapy and radiation after resection, later trials were unable to replicate the initial findings [2,11,15]. Lack of new chemo-therapeutic combinations in the immediate postresection stage has kept the 5-year overall survival from high grade astrocytomas between 10-20\% [1,3,16].

Between 30-50\% of HGG are found within the cerebral hemispheres, with the rest developing from structures like the thalamus, hypothalamus and basal ganglia [10]. Very rarely do these neoplasms originate in infratentorial structures or the spinal cord. The location of these tumors correlates highly with the initial presentation of symptoms and can include headache, early morning emesis and other signs of increased intracranial pressure, hemiplegia and focal motor deficits. Due to the aggressive nature of these tumors the time between symptoms and diagnosis is significantly shorter than with slower growing neoplasms of the CNS [14].

These patients were treated with a regimen of radiation and Temozolamide as an immediate induction therapy followed by consolidation therapy with Bevacizumab and Irinotecan all after initial resection. The rationale of this treatment was to utilize a consolidation treatment that included a topoisomerase inhibitor, Irinotecan, to bypass any remaining tumor that may be non-responsive to O6methylguanine-DNA methyltransferase (MGMT) phenotype neoplasms. Additionally, this combination of chemotherapy has been previously proven to be effective in patients treated for recurrence of HGG suggesting utility in "resistant" neoplasia and is also currently under investigation in a COG protocol $[5,14,16,19]$. Although this regimen cannot be described as uniquely novel as it is currently being investigated, this combination of therapy requires immediate consultation and cooperation of oncologists, radiation oncologists and neurosurgeons. The prospect of improved survival and performance status, even in the face of sub-total resection, is encouraging and supplicates immediate action by neurosurgeons operating on HGG in children.

HGG prognosis is largely influenced by the location of the tumor, age of the patient, and percentage of surgical resection [13]. Excluding other variables, progression free survival remains 4-6 months with an overall survival of patients with these tumors between 12-18 months [4,7]. These patients did extraordinarily well on this therapeutic regimen, surpassing expectations, with one patient having 18 months of PFS and one patient achieving CR1 at the 
time of this publication for 20 months. The treatment regimen was well tolerated without unanticipated toxicities or episodes of severe neutropenia induced by this therapy. Both patients and parents subjectively reported improved quality of life, both during and immediately following therapy. Further, both patients are still living, surpassing expectations for OS as well. The patient who relapsed has been successfully maintained on additional therapy (treated monthly with Carboplatin and Bevacizumab) without further progression for an additional 9 months with almost complete resolution of her paraplegia.

This case series only includes two patients, and thus it is difficult to generalize these findings for other patients. However, given the outcomes experienced by these patients (relative to standard outcomes), further protocols using a combined induction and consolidation approach are warranted for HGG. We are encouraged by the improvements in PFS and OS in these two patients as well as the acceptability of toxicity in this case series. Thus, while this therapeutic regimen is not novel, we highly recommend that neurosurgeons operating on young patients with HGG immediately consult oncology and radiation oncology to initiate this regimen in light of the very mild toxicity profile and high performance status of the patients presented in this case series.

\section{References}

[1] Broniscer A, Chintagumpala M, Fouladi M, Krasin M, Kocak M, Bowers D, Iacono L, et al. Temozolomide after radiotherapy for newly diagnosed high-grade glioma and unfavorable low-grade glioma in children. J Neurooncol. 76: 313-319, 2006.

[2] Finlay J, Boyett J, Yates A, Wisoff J, Milstein J, Geyer J, et al. Randomized phase III trial in childhood high-grade astrocytoma comparing vincristine, lomustine, and prednisone with the eightdrugs-in-1-day regimen. Childrens Cancer Group. J Clin Oncol. 13: 112-123, 1995.

[3] Finlay J, Zacharoulis S. The treatment of high grade gliomas and diffuse intrinsic pontine tumors of childhood and adolescence: a historical-and futuristic-perspective. J Neurooncol. 75: 253-266, 2005.

[4] Gruber M, Buster W. Temozolomide in combination with irinotecan for treatment of recurrent malignant glioma. Am J Clin Oncol. 27: 33-38, 2004.

[5] Lai A, Tran A, Nghiemphu P, Pope W, Solis O, Selch M, et al. Phase II study of bevacizumab plus temozolomide during and after radiation therapy for patients with newly diagnosed glioblastoma multiforme. J Clin Oncol. 29: 142-8, 2011.

[6] Melean G, Sestini R, Ammannati F, Papi L. Genetic insights into familial tumors of the nervous system. Am J Med Genet C Semin Med Genet. 129C: 74-84, 2004.

[7] Mueller S, Chang S. Pediatric brain tumors: current treatment strategies and future therapeutic approaches. Neurotherapeutics. 6: 570-586, 2009.

[8] Narayana A, Kunnakkat S, Chacko-Mathew J, Gardner S, Karajannis M, Raza S, et al. Bevacizumab in recurrent high-grade pediatric gliomas. Neuro Oncol. 12: 985-90, 2011.

[9] Perkins S, Rubin J, Leonard J, Smyth M, El Naqa I, Michalski J, et al. Glioblastoma in children: a single-institution experience. Int J Radiat Oncol Biol Phys 80: 1117-21, 2011.

[10] Pettorini B, Park Y, Caldarelli M, Massimi L, Tamburrini G, Di Rocco C. Radiationinduced brain tumours after central nervous system irradiation in childhood: a review. Childs Nerv Syst. 24: 793-805. 2008.

[11] Pollack I, Boyett J, Yates A, Burger P, Gilles F, Davis R, et al. The influence of central review on outcome associations in childhood malignant gliomas: results from the CCG-945 experience. Neuro Oncol. 5: 197-207, 2003.

[12] Pollack I. Brain tumors in children. N Engl J Med. 331: 1500-7, 1994.

[13] Qaddoumi I, Sultan I, Gajjar A. Outcome and prognostic features in pediatric gliomas: a review of 6212 cases from the Surveillance, Epidemiology, and End Results database. Cancer. 115: 5761-70. 2009.

[14] Reulecke B, Erker C, Fiedler B, Niederstadt T, Kurlemann G. Brain tumors in children: initial symptoms and their influence on the time span between symptom onset and diagnosis. J Child Neurol. 23: 178-183, 2008.

[15] Sposto R, Ertel I, Jenkin R, Boesel C, Venes J, Ortega J, et al. The effectiveness of chemotherapy for treatment of high grade astrocytoma in children: results of a randomized trial. A report from the Childrens Cancer Study Group. J Neurooncol. 7: 165-77, 1989.

[16] Stark-Vance V: Bevacizumab and CPT-11 in the treatment of relapsed malignant glioma. Neuro-Oncology 7: 369, 2005.

[17] Tamber M, Rutka J. Pediatric supratentorial highgrade gliomas. Neurosurg Focus. 15:14 (2): e-pub, 2003.

[18] Varley J, McGown G, Thorncroft M, Santibanez-Koref M, Kelsey A, Tricker K, et al. Germline mutations of TP53 in Li-Fraumeni families: an extended study of 39 families. Cancer Res. 57: 3245, 1997.

[19] Vredenburgh J, Desjardins A, Herndon J, Marcello J, Reardon D, Quinn J, et al. Bevacizumab plus irinotecan in recurrent glioblastoma multiforme. J Clin Oncol. 25: 4722-9, 2007

[20] Ward B, Gutmann D. Neurofibromatosis 1: from lab bench to clinic. Pediatr Neurol. 32: 221-228. 2005.

[21] Wisoff JH, Boyett JM, Berger MS, Brant C, Li H, Yates A, et al. Current neurosurgical management and the impact of the extent of resection in the treatment of malignant gliomas of childhood: a report of the Children's Cancer Group trial no. CCG-945. J Neurosurg. 89: 52-59, 1998. 